\title{
Optimal Ocean Navigation
}

\author{
C. de Wit \\ University of Technology \\ Subdep. of Mathematics \\ Delft - Netherlands.
}

Abstract.

A merchant or navy ship is to cross a wide ocean in minimum time. With the available data regarding input disturbances and system's - i.e. ship's - performance, this paper is mainly dedicated to the practical implementation of the solution of this nonlinear problem. An account of the incompleteness of the various data, while the ship's master and his officers are primarily responsible for damage to ship and cargo, the description of the solution method is given in a most plausible form, so that it is well understandable to the practical navigator.

This was one of the reasons for using the concept of timefronts. The rather frequent occurrence of conjugate points on tracks, that can be constructed on the basis of the usual necessary conditions as well as the fair possibility that such a curve without conjugate points is only time-optimal in a local sense were other arguments to make a search for the global solution of this problem by means of timefronts. 
1. Optimization criterion.

The problem of navigating a ship across a wide ocean has been an adventurous challenge throughout the centuries. In the old days the main feature of this problem was the question of feasibility without substantial damage to ship, crew and cargo. In the past 25 years however, the emphasis has been shifted to the search for an "optimal" solution in some sense.

By far the most important criterion of optimization has until now been the time itself, i.e. the trip's duration. Other criteria could be to minimize the fuel consumption or the occurrence of bad weather and high waves. Mainly on account of the lack of reliable data, these criteria have until now not been taken into study for practical application. 
2. The least time problem.

2.1. Preliminary data.

The search for a ship's least time track across an ocean needs a fair knowledge of the following things.

(1) In the first place we have the disturbing inputs, like the significant wave heights, the mean directions of wave propagation, the wind speeds and directions 21 l over the area for a timelast of at least the mean trip's duration.

(2) Secondly we need to know the system's performance, i.e. the ship's maximum speed under these weather conditions into various directions, possibly taking account of restrictions, caused by safety considerations.

Considering the weather data, the availability is restricted to a future time period of at most 72 hours. This implies that $f . i$. for a trip with a minimal duration of 6 days, the initial estimate of a least time track can only be time optimal for the first 2-to-3-days' part. Practical experience has shown, that there is little or no sense in making any assumptions for the later part.

As for the ship's performance data, figure 1 shows an example of the ship's attainable speed into various directions, when a wind of $35 \operatorname{knots}(1 \operatorname{knot}=1852 \mathrm{~m} / \mathrm{h}$ ) has been prevailing long enough and with sufficient fetch to develop a stationary field of wind waves. This polar speed graph is called the "original velocity indicatrix".

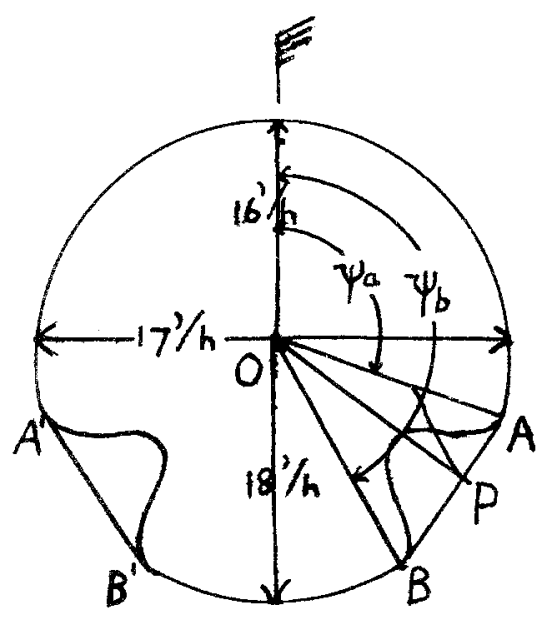

Figure 1.

Original velocity indicatrix for a ship with a nominal speed of 20 knots in waves, generated by a wind speed of 35 knots. 
The forward part of this speed graph, with ship's courses differing at most $50^{\circ}$ from the wind direction, can be determined by means of computations, based on a theory of energy dissipation. The rest of the graph has to be acquired by means of practical or at least full scale measurements, because for these cases there is no satisfactory model available.

The concave dents in the "wind-free-parts" are a result of the fact, that the apparent frequency of the waves - coming in between the beam and the stern - is rather low. With an unferourable static stability, the ship is liable to get a considerable list during several minutes without the presence of a sufficiently restoring torque. This phenomenon can only be prevented by a considerable speed reduction in that sector. With these reduced speeds however, these courses cannot be selected for a time-optimal track.

We now replace - see fig. 1 - the original velocity indicatrix by its convex envelope.

In fig. 1 the concave ares $A B$ and $A^{\prime} B^{\prime}$ are then replaced by straight Iine segments, that are tangents to the original indicatrix.

When a ship should wish to proceed as fast as possible into the direction OP, she would have to tack on the courses $\psi_{a}$ and $\psi_{b}$.

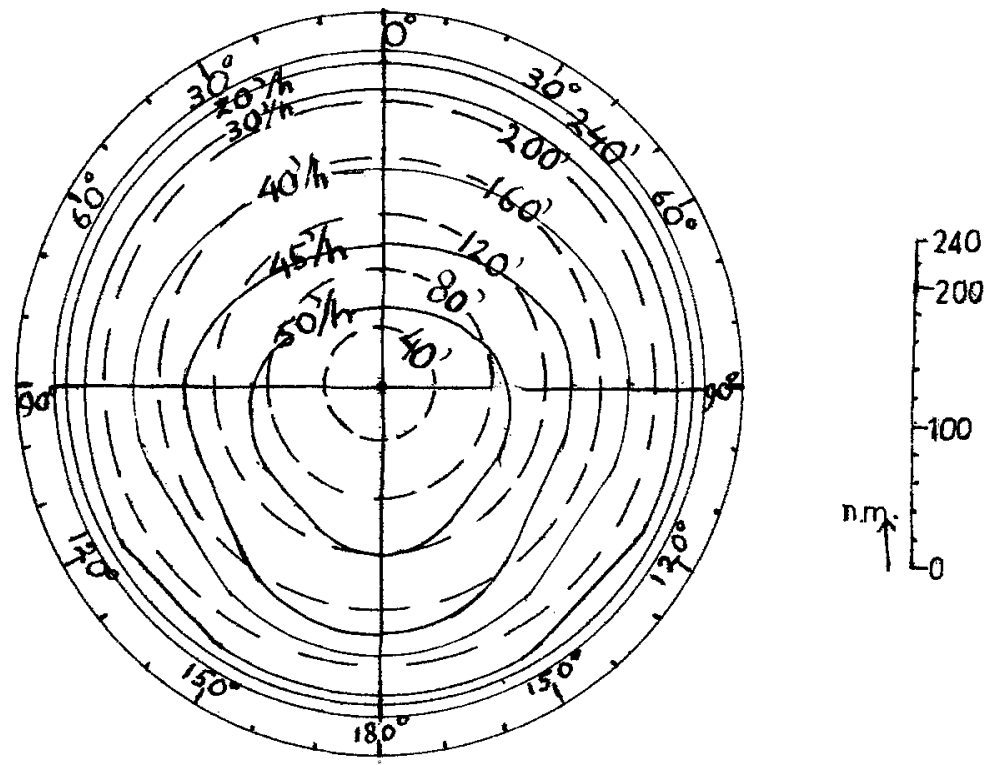

Figure 2.

Effective $12^{\text {h }}$-position indicatrices for a vessel with a nominal speed of 20 knots in waves, generated by wind velocities of $0,20,30(5) 50$ knots. 
Figure 2 shows a ship's performance in 12 hours for various courses-relative to the wind direction'- and for various wind velocities. The $12^{\text {h }}$-interval has been chosen because most weather stations broadcast weather prognoses for times with mutual intervals of 24 hours.

\subsection{Construction of subsequent timefronts.}

The construction of (the initial estimate of) a least time track can be carried out by using the concept of timefronts. A timefront $\partial_{t}$ can be defined as the boundary of the attainable region $\dot{H}_{t}$ at time $t$, when starting in $\underline{x}_{0}$ at time $t_{0}$.

The main problem now is the construction of a timefront $\partial \mathrm{H}_{k+1}$, for a time $t_{k+1}=$ $=t_{0}+(k+1) 24^{b}$, when the previous timefront $\partial \mathrm{H}_{\mathrm{k}}$ is given.

For this construction, one needs the weather maps $\mathrm{N}_{k}$ and $\mathrm{N}_{k+1}$ made up for times $t_{k}$ and $t_{k+1}$.

Figures 3 and 4 give a view of this construction.

Weather map $\mathrm{M}_{\mathrm{k}}$ shows the situation at time $t_{k}$. From timefront $\partial \mathrm{H}_{\mathrm{k}}$ as a start we first construct timefront $2 \mathrm{H}_{\mathrm{k}+\frac{1}{2}}$ for a time $t_{k}+12^{\mathrm{h}}$. To find the timefront, we need the weather situation at time $t_{k}+6^{h}$ as an average during the time interval $\left[t_{k}, t_{k+\frac{1}{2}}\right)$. Therefor we compare $M_{k}$ with $M_{k+1}$ and give $M_{k}$ a $6^{\text {h }}$-forward shift.

From $\partial_{\mathrm{k}}$ we now draw a sufficiently dense collection of line segments perpendicular to $\partial \mathrm{H}_{\mathrm{K}}$ and pointing to the outside of $\mathrm{H}_{\mathrm{K}}$. On these segments the wind directions and speeds can now be fairly estimated, i.e. derived from the directions and mutual distances of the isobars.

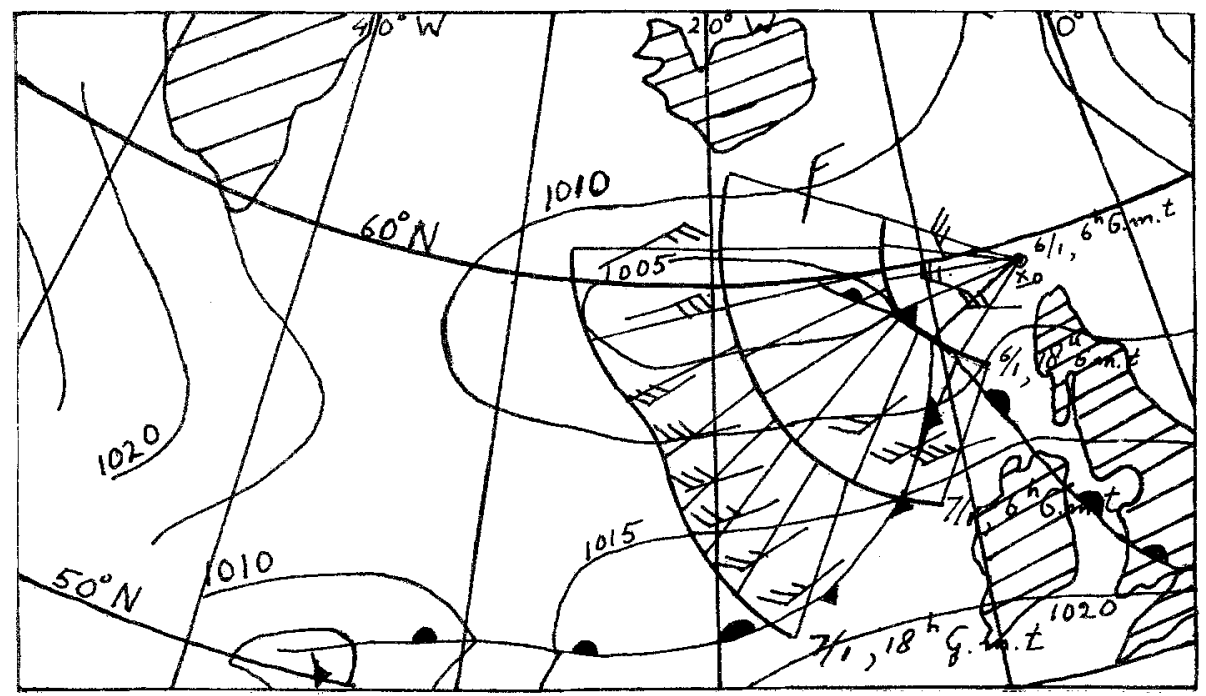

Figure 3.

Starting at $59^{\circ} \mathrm{Nh}, 5_{\mathrm{h}}^{\mathrm{o}} \mathrm{W}$ on jan. $6^{\text {th }}$, $6^{\mathrm{h}} \mathrm{G}$ G.m.t., the timefronts for jan. $6^{\text {th }}, 18^{\mathrm{h}}$ G.m.t. and for jan. $7^{\text {th }} 6^{h}$ f.m.t. have been constructed. With the estimated weather situation for jan. $7^{\text {th }}, 12^{\mathrm{h}}$ G.m.t. the timefront for jan. $7^{\text {th }}, 18^{\mathrm{h}} \mathrm{G}$.m.t. is constructed. Wind speed code: : 25 knots, : 30 knots. 


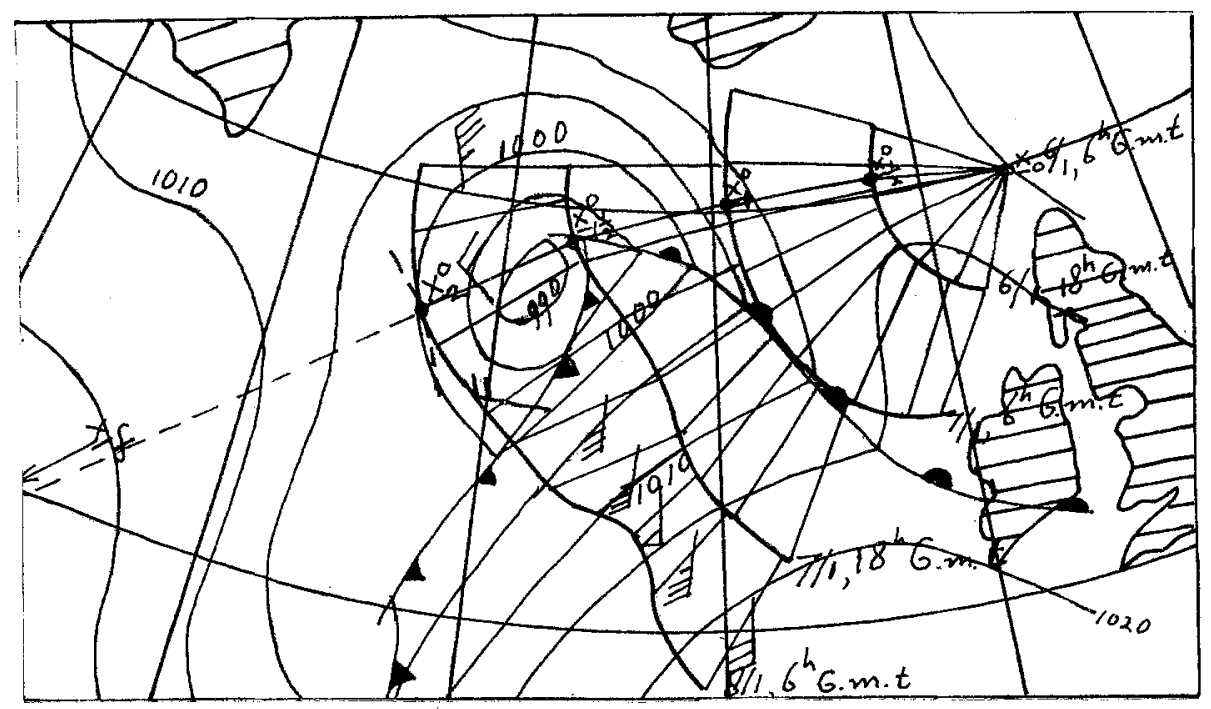

Figure 4.

Fepm the timefront of jan. $7^{\text {th }}, 18^{\text {h }}$ G.m.t. and with the use of the weather map for , jan. $8^{t h}, 0^{h}$ G.m.t. the timefront is constructed for jan. $8^{\text {th }}, 6^{h}$ G.m.t.

With the aid of the $12^{\text {h }}$-performance diagram (fig.2) the ship's ultimate position, sailing along one of these lines during 12 hours, can now be found. These points can be connected by a smooth curve, which is the relevant part of $\partial H_{k+\frac{1}{2}}$.

From this intermediate timefront, one can now obtain $\partial \mathrm{H}_{k+1}$ in the same manner, only now the time interval is $\left[t_{k}+12^{h}, t_{k}+24^{h}\right)$ with $t_{k}+18^{h}$ as the midpoint. This means that we now have to use $M_{k+1}$ with a $6^{h}$-shift backward.

\subsection{Estimation of the initial course.}

With $\underline{x}_{0}$ at time $t_{0}$ as a starting point and with the weather maps $M_{0}^{0}$, $M_{i}^{0}$ and $M_{2}^{0}$ at our disposal, we can now construct the timefronts $\partial \mathrm{H}_{\frac{1}{2}}^{0}, \partial \mathrm{H}_{i}^{0}, \partial \mathrm{H}_{1 \frac{1}{2}}^{0}, \partial \mathrm{H}_{2}^{0}$. Now when the destination $\underline{x}_{f}$ is situated outside of $\underline{H}_{2}^{0}$, we can determine the point $\underline{x}_{2}^{0}$ of the last timefront $\partial \mathrm{H}_{2}^{0}$, that is closest to $\underline{x}_{f}$ in a purely geodesic sense. From $\underline{x}_{2}^{0}$ we can then work backwards. $i . e$, we can determine $x_{k-\frac{7}{2}}^{0}$ from $x_{k}^{0}$ by drawing a Iine from $x_{k}^{0}$ perpendicular to $\partial \mathrm{F}_{k-\frac{1}{2}}^{\mathrm{O}}$ for $k=2,1 \frac{1}{2}, 1$. The initial course is then given by the vector $\underline{x}_{\frac{1}{2}}^{0}-\underline{x}_{0}$ and the ship takes the track from $\underline{x}_{0}$ via $\underline{x}_{\frac{1}{2}}^{0}$ to $\underline{x}_{1}^{0}$ as a schedule for the first 24 hours.

$1{ }_{1}$ At time $t_{1}$ the ship receives new weather information in the form of weather maps $M_{1}^{1}, M_{2}$ and $M_{3}$. Also, a position fix may give an updating $\underline{x}_{1}$ of the planned position $x_{1}^{0}$. With these new data the entire procedure is repeated, until the destination $\underline{x}_{f}$ lies 
in between $\partial \mathrm{H}_{\mathrm{k}}^{\mathrm{k}-2}$ and $\partial \mathrm{H}_{\mathrm{k}-\frac{1}{2}}^{\mathrm{k}-2}$ for some $\mathrm{k}$.

In that case we ean determine $x_{k-\frac{7}{2}}^{k-2}$ by drawing a perpendicular line from $x_{f}$ onto $\partial H_{k-\frac{1}{2}}^{k-2}$, then find $x_{k-1}^{k-2}$ aso in the same manner. 
3. Practical implementation.

The entire procedure can be carried out either by the ship's navigator, in which case one can speak of "weather navigation" or by a shore weather institute. In this last case the weather station sends a routeing advice to the ship, explains it briefly and adapts it whenever necessary. This is called "weather routeing".

Both systems have their advantages. In the case of autonomous navigation the ship's officers are better able to adapt their constructions whenever the ship's performance turns out to deviate from the expectations. On the other hand, a weather burean is able to obtain a better view on the expected weather and sea situations, as it can dispose of more meteorological information. 
4. Automation.

The author of this paper designed a computer algorithm for calculations of a least time track. (Ref. 1,2). The co-state differential equations were bypassed by using the property, that the costate vector $\mathrm{D}$ is positively proportional to the timefront's gradient. Moreover, the time-optimal course $\psi_{0}(t)$, following at a certain time $t$ from the given state $\underline{x}(t)$ and a certain co-state $\underline{p}(t)$ by $\rho \underline{\underline{p}}(t)$, where $p$ is an arbitrary positive scalar.

In areas of strong winds, high waves and consequently low ship's performances, the timefronts may overwash each other, like indicated in figure 5.

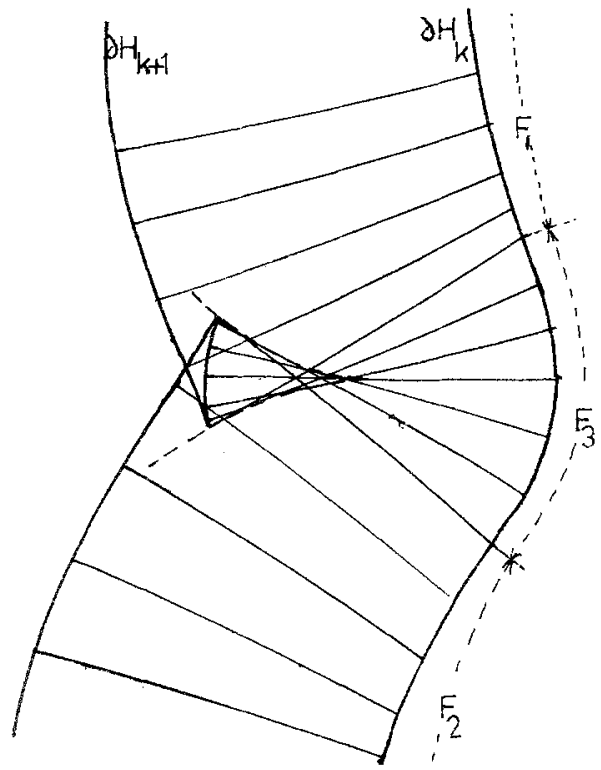

Figure 5 .

A coneave dent in $\partial \mathrm{H}_{\text {f }}$ frequently results in a splitting up of all the least time trajectories into two families of (still least time) trajectories $F_{1}$ and $F_{2}$ and a collection of trajectories, that are not even local time extremals past a certain conjugate point.

The difficulty then axises that the timefront's gradient may not exist. This difficulty was overruled by Bijlsma (Ref. 4), who determined the coefficients of the co-state differential equations of numerical differentiation.

\section{References.}

1. de Wit, C. - Mathematical Treatment of Optimal Doean Ship Routeing; Rotterdam, 1968. (Ph.D. Thesis).

2. de Wit, C. - Optimal Meteorological Ship Routeing; Report 142 s, Neth. Ship Research Centre T.N.O., Delft, 1970.

3. de Wit, C. - Progress and Development of Ocean Weather Routeing; Report 201 S, Neth. Ship Res. Centre T.N.O., Delft, 1974.

4. BijIsma, S.J. - On minimal time ship routing; Staatsdrukkerij The Hague, 1975. 